Desi gn of pol ar i zati on converter based on phot oni $c$ crystal $f$ i ber wi th ani sot ropi $c$ I at i ce core consi sting of ci rcul ar hol es

\begin{tabular}{|l|l|}
\hline 著者 & $\begin{array}{l}\text { ZHANG Zej un, TSUJ Y Yasuhi de, EGUCH Nasashi , } \\
\text { CHEN Chunpi ng }\end{array}$ \\
\hline $\begin{array}{l}\text { j our nal or } \\
\text { publ i cat i on t i t l e }\end{array}$ & Journal of the Opt i cal Soci ety of Amer i ca B \\
\hline vol une & 34 \\
\hline number & 10 \\
\hline page r ange & $2227-2232$ \\
\hline year & 2017 \\
\hline URL & ht t p: //hdl . handl e. net /10258/00010166 \\
\hline
\end{tabular}




\title{
Design of Polarization Converter Based on PCF with Anisotropic Lattice Core Consisting of Circular Holes
}

\author{
Zejun Zhang ${ }^{1},{ }^{*}$, Yasuhide TsuJI ${ }^{2}$, Masashi Eguchi ${ }^{3}$, And Chunping Chen ${ }^{1}$ \\ ${ }^{1}$ Department of Electrical, Electronics and Information Engineering, Kanagawa University, Yokohama, Japan, 221-8686 \\ ${ }^{2}$ Division of Information and Electronic Engineering, Muroran Institute of Technology, Muroran, Japan, 050-8585 \\ ${ }^{3}$ Department of Photonics System Technology, Chitose Institute of Science and Technology, Chitose, Japan, 066-8655 \\ *Corresponding author:zhang-zj17@kanagawa-u.ac.jp
}

Compiled August 30, 2017

\begin{abstract}
In this paper, we propose a novel polarization converter (PC) based on a photonic crystal fiber (PCF) with an anisotropic square lattice core consisting of circular air holes. Utilizing the core symmetry oriented at a 45-degree to the principal axes, an incident polarized light beam can be completely rotated $90^{\circ}$. Simulation results by using a vectorial finite element beam propagation method show that the polarization conversion is achieved at a device length of $119 \mu \mathrm{m}$. Additionally, an improved PC element that can provide a high-consistent Gaussian-like mode distribution to decrease the splice loss with conventional fibers has also been studied. The polarization conversion efficiency is better than $99 \%$ and the corresponding extinction ratio is better than $-20 \mathrm{~dB}$ over a wavelength range of $310 \mathbf{n m}$. ๑ 2017 Optical Society of America
\end{abstract}

OCIS codes: (060.2340) Fiber optics components; (130.5440) Polarization-selective devices.

http://dx.doi.org/10.1364/ao.XX.XXXXXX

\section{INTRODUCTION}

With the development of optical communication society, the demand for high-performance optical devices is also increasing. Polarization converters (PCs), which play an important role in polarization diversity systems, attract the interest of many researchers, and several types of PCs have also been investigated and proposed [1]-[7]. Initially, a PC based on multi-section asymmetric rib waveguides was proposed [1]. However, this kind of PC suffers from a long device length and large transition losses between alternating sections. After that, several waveguide type PCs [3]-[7] have been designed. In order to realize the optical axis rotation of a waveguide, some specific asymmetric structures have been introduced, such as off-axis double cores [3], asymmetric Si nanowire [4], bi-level tapers [5], and so on.

Photonic crystal fibers (PCFs) [8],[9], also known as microstructure fiber, are defined by a single material with a lattice of air holes running parallel to the axis. Since the air hole shape and position can be chosen with great flexibility, several unique characters can be achieved by PCF, such as endless single mode, high birefringence, absolutely single polarization transmission, etc. [9]. In recent years, high-performance optical devices using high-birefringence PCFs have been widely investigated [10][19]. With the further study of PCF, the photonic bandgap silica PCF with anisotropic material such as liquid crystal (LC), has been confirmed to have a potential for polarization conversion [13], then a number of fiber type PCs based on LC-PCF have been investigated [14]-[16]. A high tunable PC based on soft glass PCFs with an anisotropic cladding where air holes have been infiltrated with nematic liquid crystal (NLC-PCF) was reported by Hameed and Obayya [14]. After that, in order to enforce the coupling between the two fundamental polarization states, PCs with an asymmetric core have been proposed [17],[18]. In 2011, Hameed et al. proposed a PC with an Lshaped core [17]. However, the L-shaped core produces a nonGaussian mode distribution, which makes it difficult to connect with conventional single-mode fibers (SMFs). In 2016, Zhang et al. proposed a novel PC element with a symmetric core distribution by using PCFs with elliptical air holes in the core [19]. The designed single-element PC can achieve almost $100 \%$ polarization conversion with a device length of $31.7 \mu \mathrm{m}$, and both input and output ports have a Gaussian-like field distribution. Moreover, a cross-talk free PC element is obtained by adopting two single-polarization waveguides at both sides of the singleelement PC. Even the PC has a high conversion efficiency (CE), the elliptical-hole lattice core leads to low structural tolerance. Therefore, in order to actually apply it to the optical fiber communication system, high structural tolerance PCs should be discussed. 


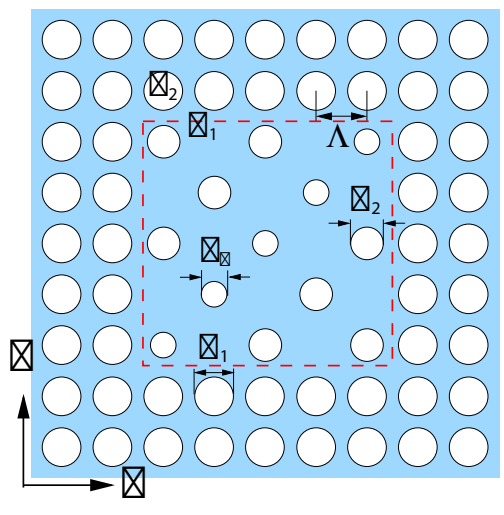

Fig. 1. Cross-sectional view of square-lattice PC with only circular air holes (the core is indicated by a red dashed frame).

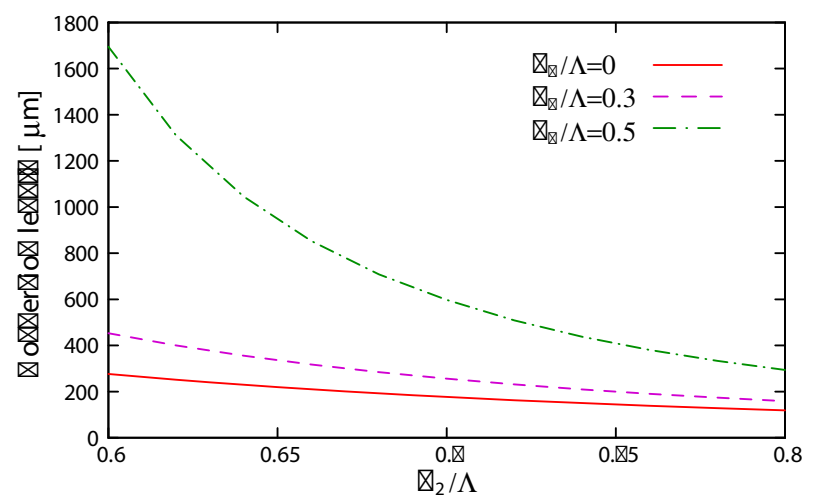

Fig. 2. Hole size dependence of conversion length for the PC in Fig. 1.

In this research we offer two kinds of square lattice PCF based PC elements with only circular air holes which has a symmetric core. The anisotropic property caused by the defects of certain holes and different hole sizes in the core can be used to realize polarization conversion. In comparison with Ref. [19], this study mainly focuses on the design of a single-element PC that can realize a Gaussian-like field distribution and has high structural tolerance at the same time. In this paper, firstly, we present our proposed PC element with a symmetric core. The core hole size dependence of the conversion length is discussed and a complete polarization conversion can be achieved with a device length of only $119 \mu \mathrm{m}$. After that, in order to obtain a high-consistent Gaussian-like mode distribution, an improved PC structure is designed. The structural parameters and light propagation behavior are discussed and illustrated in detail. Moreover, the simulation results show that the improved PC has large structural tolerance and wide wavelength bandwidth corresponding to a high CE. According to the current PCF manufacturing process, square lattice PCFs can be produced by using the stack and draw fabrication [20]. Therefore, we believe that our proposed PC element has the potential to be put into production and application in the future. In this paper, the fullvectorial finite element method (FV-FEM) [21] and finite element beam propagation method (FE-BPM) [22] have been used to estimate the effective index of the waveguide and the light propagation behavior, respectively.
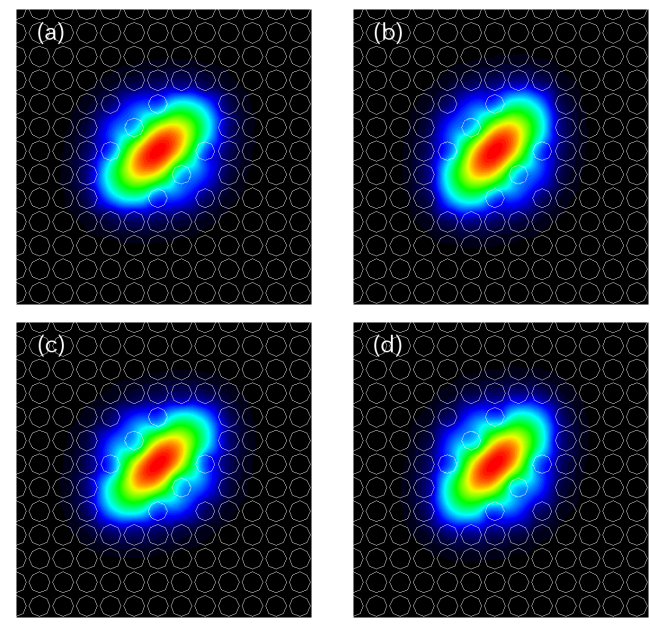

Fig. 3. Magnetic field distributions of the (a) $H_{x}$ and (b) $H_{y}$ components for the fundamental mode, and the (c) $H_{x}$ and (d) $H_{y}$ components for the $1^{\text {st }}$-higher-oder mode with $d_{1}=0.88 \Lambda$, $d_{2}=0.8 \Lambda$ and $d_{3}=0$.

\section{POLARIZATION CONVERTER WITH ONLY CIRCULAR AIR HOLES}

In this section, firstly, we illustrate our proposed single-element PC. After adjusting the hole sizes, polarization conversion is achieved at a device length of $119 \mu \mathrm{m}$. Then, an improved PC structure is given to obtain a high-consistent Gaussian-like mode distribution. The mode matching between the obtained PC fundamental mode and Gaussian field distribution is investigated in detail. For this improved PC, the light propagation behavior, structural tolerance, and wavelength dependence have been discussed.

\section{A. Design of PC with only circular air holes}

A cross-sectional view of our proposed single-element PC is shown in Fig. 1. All of the air holes are arranged in a square lattice pattern, and the core represented by a red dashed box is symmetric with respect to $y=x$. With the defects of certain air holes and different hole sizes which are expressed using $d_{2}$ and $d_{3}$, an anisotropic core is achieved. Two hybrid modes which are polarized in the $\pm 45^{\circ}$ direction with respect to $x$-axis can be transmitted in this PC with different effective indices, then polarization conversion may occur. In this study, the modal hybridness is defined as the non-dominant to dominant field ratio [15]:

$$
\text { Hybridness }=\max \left|H_{\mu}\right| / \max \left|H_{\nu}\right|,
$$

where $\mu$ and $\nu$ are $x$ and $y$ for $x$-polarized mode while $y$ and $x$ for $y$-polarized mode, respectively. Moreover, $H_{x}$ and $H_{y}$ represent the amplitudes of field components. According to the coupled mode theory, the minimum longitudinal distance at which the maximum polarization conversion occurs is referred to as the conversion length $L_{c}$ and it can be calculated by

$$
L_{c}=\frac{0.5 \lambda}{n_{\mathrm{eff}, 1}-n_{\mathrm{eff}, 2}}
$$

where $n_{\text {eff,1 }}$ and $n_{\text {eff,2 }}$ represent the effective refractive indices of the fundamental and $1^{\text {st }}$-higher-order modes, respectively.

The basic structural parameters are set as follows: the square lattice pitch is $\Lambda=0.5 \mu \mathrm{m}$, the diameter of cladding air holes is 


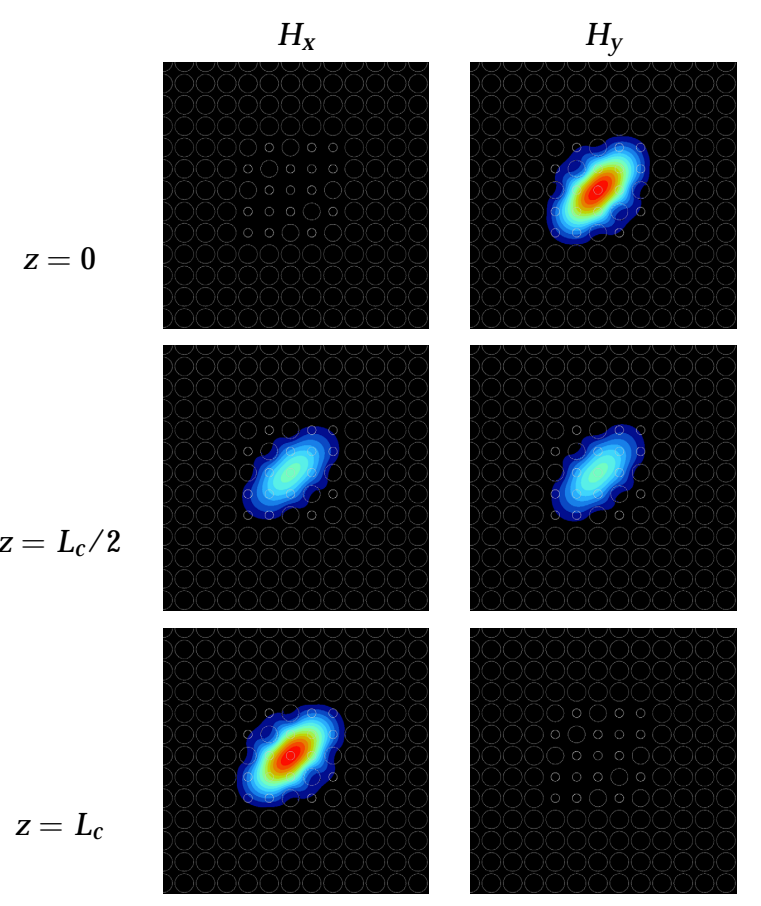

Fig. 4. Propagation behavior of an $x$-polarized incident light in the PC with $L_{c}=119 \mu \mathrm{m}$.

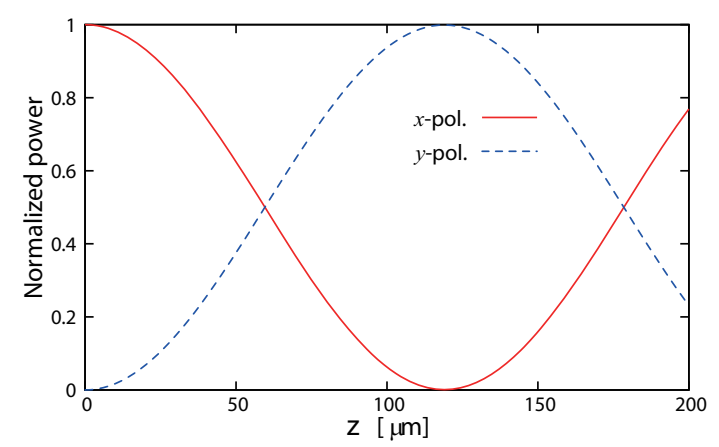

Fig. 5. Normalized power variation of the $x$-polarized incident light against the propagation distance.

$d_{1}=0.88 \Lambda$, the wavelength is set to $\lambda=1.55 \mu \mathrm{m}$, the refractive indices of silica and air are set to $n_{1}=1.45, n_{2}=1$, respectively. Since there are two kinds of air holes in the core (the respective diameters are $d_{2}$ and $d_{3}$ ), we set $d_{2}>d_{3}$ to confine light at the center of PC. The core hole size dependence of conversion length is shown in Fig. 2. We can see that the PC has a relatively short conversion length with large $d_{2}$ and small $d_{3}$. This is because with a large hole size difference between $d_{2}$ and $d_{3}$, the core birefringence is enhanced and polarization conversion can be achieved within a short device length. For example, if the core hole sizes are set to $d_{2}=0.8 \Lambda$ and $d_{3}=0$, then the corresponding conversion length is $L_{c}=119 \mu \mathrm{m}$. The magnetic field distributions of $H_{x}$ and $H_{y}$ components of for the two hybrid modes (fundamental and $1^{\text {st }}$-higher-oder modes) obtained by using the FV-FEM are shown in Figs. 3(a) to (d). It is observed that the field profiles of the dominant and non-dominant components of each mode are very similar. The maximum normalized value of $H_{x}$ is 0.99998 , based on the maximum value of
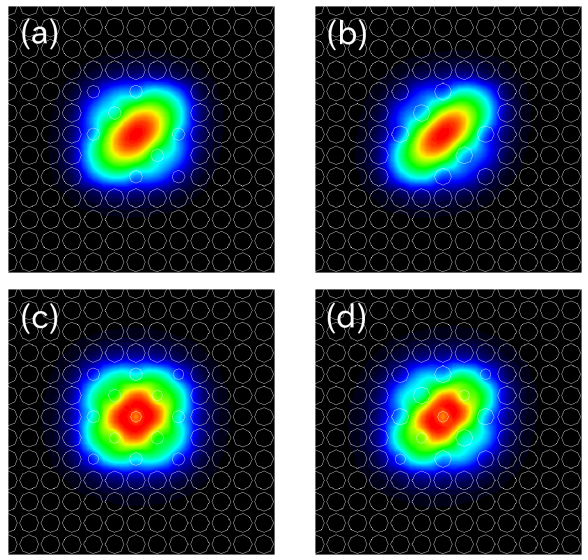

Fig. 6. Fundamental mode distributions ( $H y$ component) of PC in Fig. 1 with different core hole sizes (a) $\left(d_{2}, d_{3}\right)=$ $(0.6 \Lambda, 0),(\mathrm{b})\left(d_{2}, d_{3}\right)=(0.8 \Lambda, 0),(\mathrm{c})\left(d_{2}, d_{3}\right)=(0.6 \Lambda, 0.5 \Lambda)$, (d) $\left(d_{2}, d_{3}\right)=(0.8 \Lambda, 0.5 \Lambda)$.

Table 1. The MMR of each fundamental mode with an appropriate Gaussian field distribution ( $w$ represents spot size for each Gaussian distribution) and the corresponding conversion length $L_{c}$.

\begin{tabular}{ccccccc}
\hline & \multicolumn{2}{c}{$d_{3}=0$} & & \multicolumn{2}{c}{$d_{3}=0.5 \Lambda$} \\
\cline { 2 - 3 } \cline { 5 - 6 } \cline { 5 - 6 } & \multicolumn{2}{c}{$d_{2}$} & & \multicolumn{2}{c}{$d_{2}$} \\
\hline MMR & $91 \%$ & $88 \%$ & & $94 \%$ & $92 \%$ \\
$(w / \Lambda)$ & $(2.2)$ & $(2.1)$ & & $(2.3)$ & $(2.2)$ \\
$L_{c}[\mu \mathrm{m}]$ & 276 & 119 & & 1694 & 294 \\
\hline
\end{tabular}

the dominant component $H_{y}$ for the fundamental mode. Similarly, the hybridness of the $1^{\text {st }}$-higher-order mode is 0.99998 . Figure 4 shows the BPM simulated light propagation through this PC element. Here, the longitudinal step size is set to $1 \mu \mathrm{m}$, and the computational region of $6.25 \times 6.25 \mu \mathrm{m}$ is discretized using curvilinear hybrid edge/nodal elements [21]. An adaptive mesh [22] is used and the total unknowns are 229,990. It can be seen that an $x$-polarized incident light is completely converted into the $y$-polarized light. The power conversion along the axial direction between the two modes is given in Fig. 5, the incident light component is almost completely converted into the $y$-polarization at $z=119 \mu \mathrm{m}$. However, since both of the two modes exist in the same waveguide, we should consider the extinction ratio (ER) of PC:

$$
\mathrm{ER}=10 \log _{10} \frac{\text { Output power of } x \text {-polarized mode }}{\text { Output power of } y \text {-polarized mode }} \text {. }
$$

The BPM simulation result shows that the CE is $99.9 \%$, and ER is $-32.5 \mathrm{~dB}$ at $\lambda=1.55 \mu \mathrm{m}$.

\section{B. Improved PC}

In the previous subsection, a PC with an anisotropic lattice of circular air holes has been designed, and polarization conversion can be achieved within a short device length. However, according to the BPM simulation result, we note that an oblique elliptical mode distribution exists at the input and output ports 


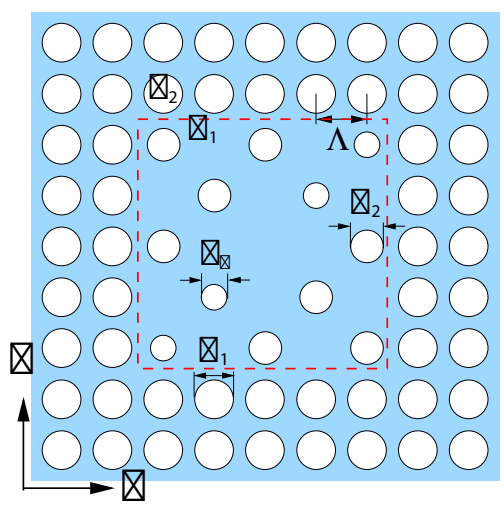

Fig. 7. Cross-section view of an improved PC.

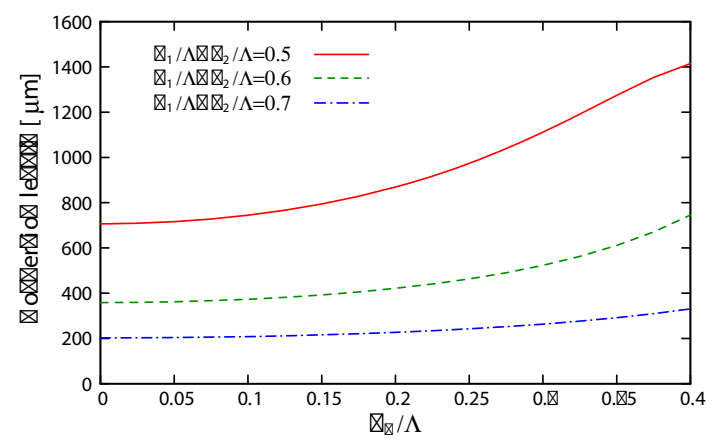

Fig. 8. Hole size dependence of conversion length for the PC in Fig. 7.

which may cause a large insertion loss with conventional fibers. Therefore, taking into account the applicability of the PC in an optical communication system, it is necessary to improve the geometry of device and optimize the structural parameters to obtain a Gaussian-like mode distribution. In this study, we measure the mode matching ratio (MMR) between the fundamental mode and a Gaussian field distribution using the following overlap integral:

$$
\operatorname{MMR}=\frac{\left|\int \phi(x, y) g(x, y) d x d y\right|^{2}}{\int|\phi(x, y)|^{2} d x d y \int|g(x, y)|^{2} d x d y}
$$

where $\phi(x, y)$ is the field distribution of fundamental mode, the Gaussian field distribution is represented by the $g(x, y)$ which is set to be $g(x, y)=\exp \left(-\left(x^{2}+y^{2}\right) / \delta^{2}\right)$, and $\delta$ is the standard deviation of $g(x, y)$. Moreover, the spot size of a Gaussian field distribution (illustrated by $w$ ) is the diameter at which the light intensity $|g|^{2}$ drops to $1 / e$ of its maximum value. The spot size can be calculated by $w=2 \sqrt{2} \delta$. The maximum value of MMR between an obtained fundamental mode and an appropriate Gaussian field distribution can be obtained by adjusting the value of $\delta$.

Figures 6 (a)-(d) illustrate the magnetic field distributions of fundamental mode (PC in Fig. 1) with $\left(d_{2}, d_{3}\right)=$ $(0.6 \Lambda, 0),(0.8 \Lambda, 0),(0.6 \Lambda, 0.5 \Lambda)$ and $(0.8 \Lambda, 0.5 \Lambda)$, respectively. Additionally, Table 1 illustrates the maximum MMR of each fundamental mode with an appropriate Gaussian field distribution (the corresponding spot size $w / \Lambda$ is illustrated under each MMR) and the corresponding conversion length. We note that although the PCs with a large $d_{2}$ have a short conversion length, the corresponding MMRs are relatively low. Moreover, a small
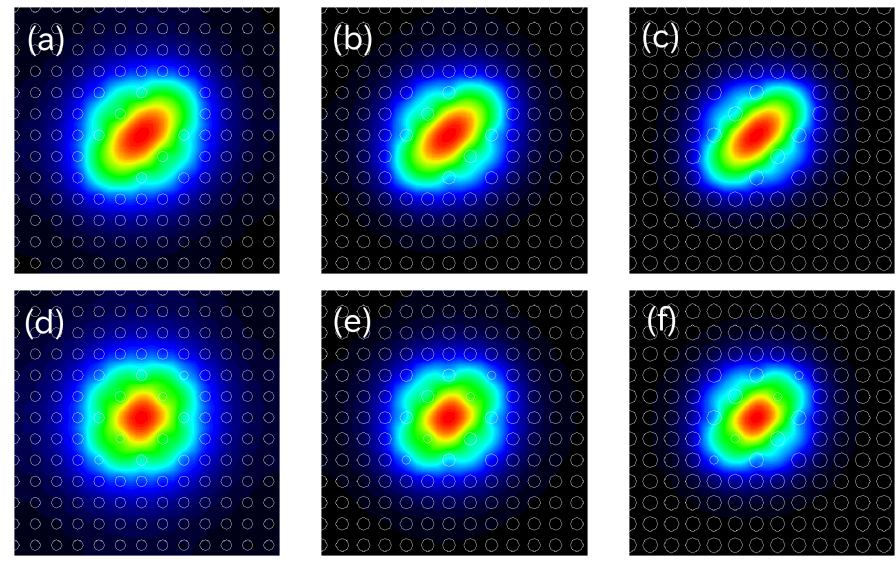

Fig. 9. Fundamental mode distributions ( $H y$ component) of PC in Fig. 1 with different core hole sizes (a) $\left(d_{1}=d_{2}, d_{3}\right)=$ $(0.5 \Lambda, 0)$, (b) $\left(d_{1}=d_{2}, d_{3}\right)=(0.6 \Lambda, 0),(\mathrm{c})\left(d_{1}=d_{2}, d_{3}\right)=$ $(0.7 \Lambda, 0),(\mathrm{d})\left(d_{1}=d_{2}, d_{3}\right)=(0.5 \Lambda, 0.4 \Lambda),(\mathrm{e})\left(d_{1}=d_{2}, d_{3}\right)=$ $(0.6 \Lambda, 0.4 \Lambda),(\mathrm{f})\left(d_{1}=d_{2}, d_{3}\right)=(0.7 \Lambda, 0.4 \Lambda)$.

Table 2. The MMR of each fundamental mode with an appropriate Gaussian field distribution ( $w$ represents spot size for each Gaussian distribution) and the corresponding conversion length $L_{c}$.

\begin{tabular}{|c|c|c|c|c|c|c|}
\hline & \multicolumn{3}{|c|}{$d_{3}=0$} & \multicolumn{3}{|c|}{$d_{3}=0.4 \Lambda$} \\
\hline & \multicolumn{3}{|c|}{$d_{1}=d_{2}$} & \multicolumn{3}{|c|}{$d_{1}=d_{2}$} \\
\hline & $0.5 \Lambda$ & $0.6 \Lambda$ & $0.7 \Lambda$ & $0.5 \Lambda$ & $0.6 \Lambda$ & $0.7 \Lambda$ \\
\hline MMR & $94 \%$ & $93 \%$ & $91 \%$ & $96 \%$ & $95 \%$ & $93 \%$ \\
\hline$(w / \Lambda)$ & (2.9) & (2.6) & $(2.4)$ & (3.0) & (2.6) & $(2.4)$ \\
\hline$L_{\mathcal{C}}[\mu \mathrm{m}]$ & 706 & 358 & 202 & 1394 & 745 & 331 \\
\hline
\end{tabular}

$d_{3}$ in the core leads to a short conversion length, however, the mode shape spreads along the oblique direction. Consequently, we consider removing an air hole at the center of core to improve the mode shape while maintaining a large anisotropic property.

The improved PC structure is illustrated in Fig. 7. The core without a center air hole not only suppresses the oblique field spreading but also reduces the reflection loss at the butt connection. In order to achieve Gaussian-like mode distribution, we reduce the effective index difference between the core and cladding region by setting the hole sizes to $d_{1}=$ $d_{2}$. The hole size dependence of conversion length is shown in Fig. 8. Since the core birefringence is in proportion to the hole size difference between $d_{2}$ and $d_{3}$, for a fixed $d_{3}$, PC with larger hole sizes $d_{2}$ has a large birefringence and a short conversion length. For a small $d_{2}$, the conversion length has a significant variation with different $d_{3}$. Especially, for $d_{3}=d_{2}$, the core is isotropic and the conversion length is infinite. Here, we illustrate the magnetic field distribution of the fundamental modes with $\left(d_{1}=d_{2}, d_{3}\right)=$ $(0.5 \Lambda, 0),(0.6 \Lambda, 0),(0.7 \Lambda, 0),(0.5 \Lambda, 0.4 \Lambda),(0.6 \Lambda, 0.4 \Lambda)$, and $(0.7 \Lambda, 0.4 \Lambda)$, respectively, as shown in Figs. 9 (a)-(f). Table 2 shows the MMR of each fundamental mode with an appropriate Gaussian field distribution $(w / \Lambda$ is the corresponding spot size) and the corresponding conversion length. For $d_{3}=0$, 


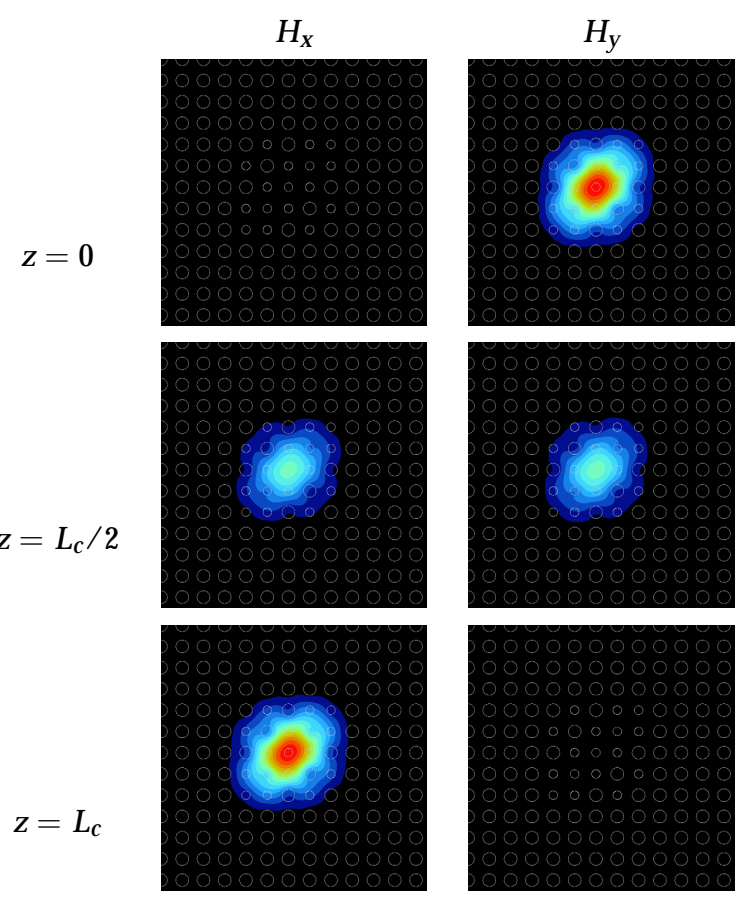

Fig. 10. Propagation behavior in the PC with $L_{c}=745 \mu \mathrm{m}$.

Table 3. CE versus the deviation for each hole size at different deviation level.

\begin{tabular}{ccccc}
\hline \multirow{2}{*}{ Deviation level } & \multicolumn{2}{c}{ Cladding } & & \multicolumn{2}{c}{ Core } \\
\cline { 2 - 2 } \cline { 5 - 5 } \cline { 5 - 5 } & $d_{1}$ & & $d_{2}$ & $d_{3}$ \\
\hline$-1.5 \%$ & $99.9 \%$ & & $98.8 \%$ & $99.8 \%$ \\
$-1.0 \%$ & $99.9 \%$ & & $99.4 \%$ & $99.8 \%$ \\
$1.0 \%$ & $99.9 \%$ & & $99.4 \%$ & $99.8 \%$ \\
$1.5 \%$ & $99.9 \%$ & & $98.8 \%$ & $99.7 \%$ \\
\hline
\end{tabular}

the PC has a high MMR with a smaller $d_{1}$ and $d_{2}$, however, due to the weak light confinement and low core birefringence, the PC has a longer conversion length. On the contrary, by adopting $d_{3}=0.4 \Lambda$, the oblique field distribution can be suppressed and a higher MMR is obtained. The BPM simulated light propagation behavior of the PC with $d_{1}=d_{2}=0.6 \Lambda$ and $d_{3}=0.4 \Lambda$ is illustrated in Fig. 10. The calculated conversion length is $L_{c}=745 \mu \mathrm{m}$. Here, the longitudinal step size is set to $1 \mu \mathrm{m}$, the computational region is still $6.25 \times 6.25 \mu \mathrm{m}$, and the total unknowns are 342,027 . When an $x$-polarized light beam is launched into the PC, it completely converts into the $y$-polarized light at $z=L_{c}$. Additionally, Fig. 11 gives the power conversion along the axial direction between the two modes. At $z=L_{c}$, the $\mathrm{CE}$ is $99.9 \%$ and the corresponding $\mathrm{ER}$ is $-32.4 \mathrm{~dB}$. Additionally, the insertion loss (IL) is defined as $\operatorname{IL}(\mathrm{dB})=-10 \log _{10}\left(P_{y} / P_{\text {input }}\right)$ with $P_{\text {input }}$ being the input power and $P_{y}$ being the power of input $y$-polarized component [7]. The calculated IL is $0.004 \mathrm{~dB}$. By contrast with BPM simulated results in the previous subsection, a high-consistent Gaussian-like mode is achieved.

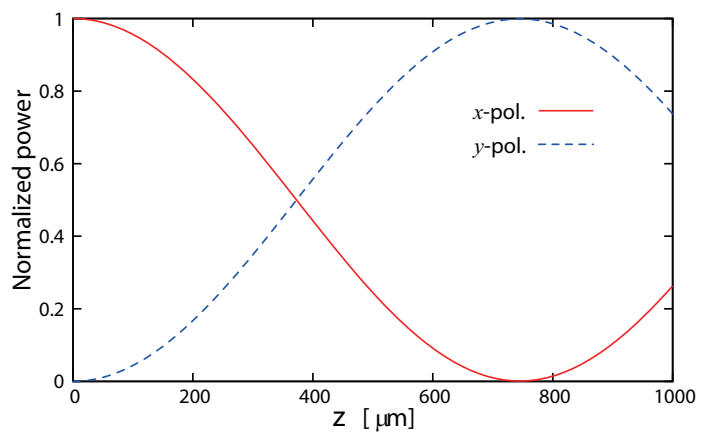

Fig. 11. Normalized power variation of the $x$-polarized incident light against the propagation distance with the device length is $L_{c}=745 \mu \mathrm{m}$.

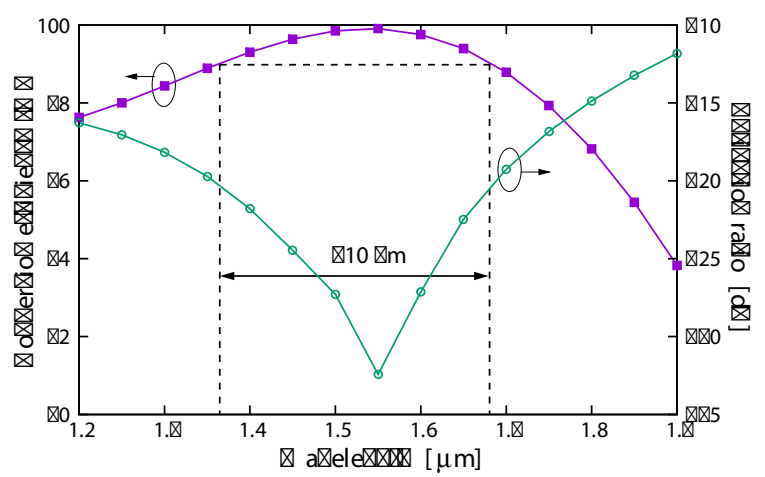

Fig. 12. Polarization conversion efficiency and extinction ratio for $x$-polarized to $y$-polarized mode conversion as a function of wavelength.

\section{Structural Tolerance and Wavelength Dependence}

So far, we have designed a simple-element section PC element with only circular air holes. Considering the fabrication technology of PCF and its application in optical communication systems, the structural tolerance and wavelength dependence should be considered. Here, we consider a PC element which has the same structural parameters mentioned in the subsection $\mathrm{B}$, i.e., the cladding hole and large core hole sizes are set with the same dimensions of $d_{1}=d_{2}=0.6 \Lambda$, the small core hole size is $d_{3}=0.4 \Lambda$, and the device length is fixed to $L=745 \mu \mathrm{m}$.

Table 3 shows the CE of PC versus the deviation for respective hole size of each region. In our investigation process, each hole size of the PC has the same variation extent, such as if only the large core holes (diameter is denoted by $d_{2}$ ) are expanded $1.5 \%$, then at the device length $L=745 \mu \mathrm{m}$, the CE of PC will descend to $98.8 \%$. This is because the effective index variations of fundamental and $1^{\text {st }}$-higher-order modes are caused by the change of hole size. Then the varied structure corresponds to a new complete conversion length and the CE of varied PC is slightly declined at the fixed device length. However, all of the air hole sizes are varied randomly during the fabrication process, some hole sizes become larger while some hole sizes turn smaller. And thus the random variation of the overall air holes lead to a relatively small effect on the effective index of the fundamental mode. According to Ref. [19], the CE of an actually obtained PC element will be higher than that of the PC hole size variations with the same tendency. Therefore, our simulation results in Table 3 give the worse CE with different hole size 
deviation levels. We note that the $\mathrm{CE}$ is sensitive to the large core hole size $d_{2}$, while the CE is almost $99 \%$ when $d_{2}$ varies within $\pm 1.5 \%$ (about $4.5 \mathrm{~nm}$ ). This structural tolerance is much larger than the PC element with elliptical holes in [19] whose CE descends to $92 \%$ when the major axis of its elliptical core hole decreases only $0.1 \%$ (about $0.8 \mathrm{~nm}$ ).

In addition, in this research, the CE is effected by the difference between determined device length $L$ and the conversion length of the two hybrid modes. The phase difference of these two modes can be given by $\Delta \theta=\left(\beta_{1}-\beta_{2}\right) L$, where $\beta_{1}$ and $\beta_{2}$ are the propagation constants of fundamental and $1^{\text {st }}$ higher-order modes, respectively. When the operation wavelength is $1.55 \mu \mathrm{m}$, since the conversion length is equal to the device length, then $\Delta \theta=\pi$, and the CE is almost $100 \%$. However, if the phase difference $\Delta \theta$ is not equal to $\pi$, the $C E$ will decrease. According to our simulation results, the difference between the two propagation constants has a low wavelength dependence, and a short device length $L$ leads to a small deviation of $\Delta \theta$ from $\pi$, that is, a high CE with a wide-band is achieved. In order to confirm the wide-band transmission, the wavelength dependence of our proposed PC has also been discussed, as illustrated in Fig. 12. The CE of the PC is better than $99 \%$ within a wavelength range of 1.37 to $1.68 \mu \mathrm{m}$ (about 310nm), and the corresponding ER is better than $-20 \mathrm{~dB}$. The bandwidth of this PC element is almost twice that in [19].

\section{CONCLUSION}

In this paper, we proposed and designed a novel PCF based PC element with an anisotropic lattice of circular air holes. High CE of our proposed PC which can be up to $99.9 \%$ is achieved with a short device length of $119 \mu \mathrm{m}$. In addition, an improved PC structure is proposed to obtain a high-consistent Gaussianlike mode distribution. The maximum MMR of the improved PC with a Gaussian mode distribution is $95 \%$ and the CE is better than $99.9 \%$ at a device length of $745 \mu \mathrm{m}$ with a high ER of $-32.4 \mathrm{~dB}$. Moreover, considering the practical application and the fabrication technology of PCF, the structural tolerance and wavelength dependence of the PC element have been discussed in detail. Since there are only two kinds of circular air holes in our proposed single-element PC, and with the continuous improvement of the manufacturing technique at present, we believe that this kind of PC has a great practical potential for optical communication systems in the future.

\section{ACKNOWLEDGMENT}

The authors would like to thank K. Ichikawa for his valuable work.

\section{REFERENCES}

1. Y. Shani, R. Alferness, T. Koch, U. Koren, M. Oron, B. I. Miller, and M. G. Young, gPolarization rotation in asymmetric periodic loaded rib waveguides, h Appl. Phys. Lett., 59, 1278-1280 (1991).

2. T. Tsuchizawa, K. Yamada, H. Fukuda, T. Watanabe, J. Takahashi, M. Takahashi, T. Shoji, E. Tamechika, S. Itabashi, and H. Morita, "Microphotonics devices based on silicon microfabrication technology," IEEE J. Sel. Topics Quantum Electron. 11, 232-240 (2005).

3. H. Fukuda, K. Yamada, T. Tsuchizawa, T. Watanabe, H. Shinojima, and S. Itabashi, "Silicon photonic circuit with polarization diversity," Opt. Express, 16, 4872-4880 (2008).

4. Z. Wang and D. Dai, "Ultrasmall Si-nanowire-based polarization rotator," J. Opt. Soc. Am. B, 25, 747-753 (2008).
5. J. Zhang, M. Yu, G. Lo and D. Kwong, "Silicon-waveguide-based mode evolution polarization rotator," J. Sel. Top. Quantum Electron., 16, 53$60(2010)$.

6. L. Liu, Y. Ding, K. Yvind and J. M. Hvam, "Silicon-on-insulator polarization splitting and rotating device for polarization diversity circuits," Opt. Express, 19, 12646-12651 (2011).

7. Y. Xu, J. Xiao and X. Sun, "A compact hybrid plasmonic polarization rotator for silicon-based slot waveguides," IEEE Photon. Technol. Lett., 26, 1609-1612 (2014).

8. J. C. Knight, T. A. Birks, P. St. J. Russell, and D. M. Atkin, "All-silica single-mode optical fiber with photonic crystal cladding," Opt. Lett., 21, 1547-1549 (1996).

9. P. St. J. Russell, "Photonic-crystal fibers," J. Ligthwave Technol., 24, 4729-4749 (2006).

10. Z. Zhang, Y. Tsuji and M. Eguchi, "Design of polarization splitter with single-polarized elliptical-hole core circular-hole holey fibers," IEEE Photon. Technol. Lett., 26, 541-543 (2014).

11. Z. Zhang, Y. Tsuji, and M. Eguchi, "Study on crosstalk-free polarization splitter with elliptical-hole core circular-hole holey fibers," J. Lightw. Technol., 32, 3956-3962 (2014).

12. Z. Zhong, Z. Zhang, Y. Tsuji, and M. Eguchi, "Study on crosstalk-free polarization splitter based on square lattice single-polarization photonic crystal fibers," J. Quantum Electron., 52, 7000107 (2016).

13. L. Scolari, T. Alkeskjold, J. Riishede, A. Bjarklev, D. Hermann, A. Anawati, M. Nielsen, and P. Bassi, "Continuously tunable devices based on electrical control of dual-frequency liquid crystal filled photonic bandgap fibers," Opt. Express, 13, 7483-7496 (2005).

14. M. F. O. Hameed and S. S. A. Obayya, "Analysis of polarization rotator based on nematic liquid crystal photonic crystal fiber," J. Lightw. Technol., 28, 806-815 (2010).

15. M. F. O. Hameed and S. S. A. Obayya, "Polarization rotator based on soft glass photonic crystal fiber with liquid crystal core," J. Lightw. Technol., 29, 2725-2731 (2011).

16. M. F. O. Hameed and S. S. A. Obayya, "Ultra short slica liquid crystal photonic crystal fiber polarization rotator," Opt. Lett., 29, 2725-2731 (2011).

17. M. F. O. Hameed, S. S. A. Obayya, and H. A. El-Mikati, "Passive polarization converters based on photonic crystal fiber with L-shaped core region," J. Ligthw. Technol., 30, 283-289 (2011).

18. M. F. O. Hameed, A. M. Heikal, and S. S. A. Obayya, "Novel passive polarization rotator based on spiral photonic crystal fiber," J. Ligthw. Technol., 25, 1578-1581 (2013).

19. Z. Zhang, Y. Tsuji, and M. Eguchi, "Design of cross-talk-free polarization converter based on square-lattice elliptical-hole core circular-hole holey fibers," J. Opt. Soc. Am. B, 33, 1808-1814 (2016).

20. D. Pysz, I. Kujiwa, R. Stepień, M. Klimczak, A. Filipkowski, M. Franczyk, L. Kociszewski, J. Buźniak, K. Haraśny and R. Buczyński, "Stack and draw fabrication of soft glass microstructured fiber optics," Bull. Pol. Acad. Sci. Tech. Sci. , 62, 667-682 (2014).

21. M. Koshiba and Y. Tsuji, "Curvilinear hybrid edge/nodal elements with triangular shape for guidedwave problems," J. Ligthw. Technol., 18, 737-743 (2000).

22. Y. Tsuji and M. Koshiba, "Adaptive mesh generation for full-vectorial guided-mode and beam propagation solutions," J. Sel. Topics Quantum Electron., 6, 163-169 (2000). 